\title{
Identifying and managing patients with delirium in acute care settings
}

\section{Penny Bond and Karen Goudie discuss a programme that aims to improve care for older people by introducing a delirium toolkit}

Correspondence

penny.bond@nhs.net

Penny Bond is improvement support team leader

Karen Goudie is clinical adviser

Both at Healthcare Improvement Scotland, Glasgow

Date of submission July 162015

Date of acceptance

September 212015

Peer review

This article has been subject to double-blind peer review and checked using antiplagiarism software

Author guidelines journals.rcni.com/r/ nop-author-guidelines

\begin{abstract}
Delirium is an acute medical emergency affecting about one in eight acute hospital inpatients. It is associated with poor outcomes, is more prevalent in older people and it is estimated that half of all patients receiving intensive care or surgery for a hip fracture will be affected. Despite its prevalence and impact, delirium is not reliably identified or well managed.

Improving the identification and management of patients with delirium has been a focus for the national improving older people's acute care work programme in NHS Scotland. A delirium toolkit has been developed,
\end{abstract}

which includes the 4AT rapid assessment test, information for patients and carers and a care bundle for managing delirium based on existing guidance. This toolkit has been tested and implemented by teams from a range of acute care settings to support improvements in the identification and immediate management of delirium.

\section{Keywords}

acute care, delirium, medical emergency, older people, patient care bundles, toolkit

HEALTHCARE IMPROVEMENT Scotland (HIS) has been leading a national improvement programme focused on older people's acute care since April 2012 using a collaborative approach with multidisciplinary teams in acute hospital settings across Scotland. The programme supports improvements in two critical areas:

- Identification and management of frailty. - Identification and management of delirium. The decision to focus on these areas was informed by a review of literature and themes emerging from national inspection reports of older people's acute care. Feedback was taken from stakeholders including colleagues from the Scottish Delirium Association, NHS Education for Scotland and academic and clinical colleagues across Scotland. At the end of a consultation with NHS board colleagues, all 14 NHS boards nominated at least one local test site and team to take part in the work to improve the understanding and management of delirium. Details of the work to identify frailty and ensure rapid comprehensive geriatric assessment in four NHS boards have been published by HIS (2014a). This article addresses the programme's handling of delirium.

\section{Delirium}

Delirium, or acute confusional state, is a medical emergency. Statistics suggest that its prevalence on medical wards in hospitals is about 20-30\%, while as many as $50 \%$ of people having hip surgery may develop delirium (National Institute for Health and Care Excellence (NICE) 2010, Inouye et al 2014). People who develop delirium are more at risk of hospital-acquired complications, such as falls and pressure ulcers and are more likely to be admitted to long-term care (NICE 2010) and have increased mortality (Travers et al 2013).

Specific factors can significantly increase the risk of developing delirium including dementia (Fong et al 2015), dehydration, multiple medications, infection and sensory impairment. People with delirium stay two or three times longer in hospital and are also more likely to experience further 
cognitive decline including dementia (Fong et al 2015). Delirium is a recognised problem in older people that is often overlooked or misdiagnosed and is distressing to individuals and their families and carers.

\section{Aim and approach}

The programme aimed to improve the quality and experience of care for older people and their families by supporting local teams to undertake small tests of changes that would improve identification and management of delirium. In agreed test sites the initial aim was to assess $95 \%$ of patients over the age of 65 for delirium.

A collaborative approach was adopted that included:

Developing tools and resources.

- Supporting test sites to develop ways to record and report their progress.

Sharing findings between local teams.

\section{Tools and resources}

HIS worked closely with the Scottish Delirium Association and other colleagues across Scotland to design, test and adapt a range of tools and resources to assess, manage and review patients with delirium. The two main tools that were tested were the 4 'As' Test (4AT) (MacLullich et al 2014) and the TIME delirium care bundle (HIS 2014b). The 4AT (Table 1) is a straightforward and effective means of screening for delirium (Bellelli et al 2014). The TIME delirium care bundle (Table 2) sets out critical actions for the period immediately after delirium is identified.

The delirium care bundle (Table 2) uses the acronym 'TIME' - Think/Triggers, Investigate/ Intervene, Manage, Engage/Explore. It acts as a guide for staff to think about triggers for delirium, investigate underlying causes, implement an appropriate management plan and engage the patient and family members. The bundle builds on the Scottish Delirium Association's evidence-based delirium pathway (HIS 2014b) and NICE

(2010) guidance.

HIS also looked at giving and receiving care during an episode of delirium. The resulting report (HIS 2013) focused on the experience of patients, families and staff and highlighted the importance of a person-centred approach to care. The technique of emotional touchpoints (Dewar et al 2010) was used to investigate individual experiences. The technique focuses on particular points in the experience (touchpoints) and asks people to select from a range of emotional words that sum up what it felt like for them. Themes from the work informed the development of the TIME bundle, specifically changing the 'E' part of the bundle from 'explain to families' to 'engage with families', ensuring families are asked whether their relative's behaviour is usual for them. The focus of the bundle is to support the identification and immediate management of delirium.

\section{Table 1 The 4AT}

\section{Alertness}

This includes patients who may be markedly drowsy, for example, difficult to rouse and/or obviously sleepy during assessment or agitated/hyperactive. Observe the patient. If asleep, attempt to wake with speech or gentle touch on the shoulder. Ask the patient to state their name and address to assist rating

Normal (fully alert, but not agitated, throughout assessment)

Mild sleepiness for $<10$ seconds after waking, then normal

Clearly abnormal

Abbreviated Mental Test (AMT4)

Age, date of birth, place (name of the hospital or building), current year

No mistakes

1 mistake

2 or more mistakes/untestable

2

Attention

Score

Ask the patient 'Please tell me the months of the year in backwards order, starting at December.' To assist initial understanding one prompt of 'What is the month before December?' is permitted

Achieves 7 months or more correctly

$<7$ months or refuses to start

Untestable (cannot start because unwell, drowsy, inattentive)

Acute change or fluctuating course

Evidence of significant change or fluctuation in: alertness, cognition, other mental function, for example, paranoia, hallucinations, arising over the past two weeks and still evident in the past 24 hours

4AT score: 4 or above: possible delirium +/- cognitive impairment. 1-3: possible cognitive impairment. 0 : delirium or severe cognitive impairment unlikely

(MacLullich et al 2014, reproduced with permission) (C)2011-2014 MacLullich, Ryan, Cash 
The bundle is not intended to be a comprehensive care package, however, but rather a guide to thinking about and initiating care planning.

Over the course of the programme HIS worked with a wide range of clinicians, organising continuing professional development accredited national and local events, organising cross-site visits and hosting online improvement clinics, WebEx and conference calls. Teams were able to network, share learning and explore examples of good practice. Feedback highlighted that participants found these approaches useful as a way to promote understanding, share and validate ideas and plan their next steps:
'The word “delirium” didn't mean much before but awareness of frailty and delirium has increased hugely. The impact (of delirium) was not previously appreciated. It is now' (senior charge nurse).

'The programme has highlighted the importance of delirium to managers so we now have the resources to address it at the level of the individual patient' (dementia nurse consultant).

'In 2012, there was not much awareness of delirium and no structured assessment process. Now, it's becoming embedded' (lead nurse).

'Working in collaboration has really helped raise the profile of delirium across Scotland' (consultant geriatrician).

\section{Table 2 TIME delirium care bundle}

\section{Initiate TIME within two hours}

Think - exclude and treat possible triggers

National Early Warning Score

T Blood glucose

Medication history (identify new medications/change of dose/medication recently stopped)

Pain review (Abbey pain scale)

Assess for urinary retention

Assess for constipation

\section{Investigate and intervene to correct underlying causes}

I Assess hydration and start fluid balance

Bloods (full blood count, urea and electrolytes, calcium, liver function tests,

C-reactive protein, magnesium, glucose)

Look for symptoms/signs of infection (skin, chest, urine, central nervous system) and perform appropriate cultures/imaging depending on clinical assessment (see Sepsis Six)

Electrocardiogram (acute coronary syndrome)

\section{Management plan}

M Initiate treatment of all underlying causes

Engage and explore (complete within two hours or if family/carers not present within 24 hours)

E Engage with patient, family and carers - explore if this is usual behaviour. How would you like to be involved?

Explain diagnosis of delirium to patient, family and carers (use delirium leaflet) Document 4AT score

\section{\begin{tabular}{l|l|l} 
Assessed/sent & Results seen Abnormality found
\end{tabular}}


While the initial focus of the work was on the identification and management of delirium it became apparent that there was also a need to raise awareness more generally and improve knowledge and skills relating to delirium. Educating staff about delirium can contribute to early recognition in older people (Varghese et al 2014).

Colleagues at NHS Education for Scotland developed a range of learning resources to enhance staff knowledge and understanding of delirium. The primary learning resources consist of two modules, which are accessible by registering on learnPro NHS (http://nhs.learnprouk.com).

The first module, An Introduction to Delirium, provides baseline knowledge and skills for all staff working in health and social care settings, while the second module, Delirium: Prevention, Management and Support, aims to improve the knowledge and skills of healthcare professionals working across all care sectors.

\section{Testing changes and reporting progress}

Using approaches such as the Model for Improvement (Langley et al 2009) and sharing learning played an important part in engaging staff. Testing changes on a small scale so that they could be easily refined made the programme more manageable for busy wards. When test sites began to collect data showing the effect on patient outcomes, staff could see the benefits of the changes. Test site teams have shared their results and learning regularly through WebEx calls and national and local improvement events.

A process for collecting data each month using a flash report template helped teams record their progress, improvements and changes achieved, for example, the number of patients assessed for delirium, changes in the number of falls or effect on length of stay. Sharing these reports online and at events allowed local teams to keep up to date and discuss progress.

Data being gathered by local teams has shown how outcomes for patients are beginning to improve. Improvements reported by individual test sites include an increase in the number of patients being assessed for delirium, a reduction in the number of patients who fall and a reduced mean length of stay.

Changes in clinical practice developed by the initial test sites are now being spread to other wards, departments and hospitals, for example, extending use of 4AT, inclusion of delirium assessment in pathways for deteriorating patients and introduction of delirium education as part of inductions for new staff.
While the focus of the work has been on the identification and management of delirium, the programme has created opportunities to improve the quality of care for older people more broadly.

Elements of the TIME bundle ensure that issues such as nutrition, pain and skin integrity are all considered. Getting these fundamental elements right consistently helps improve older people's experience of care as a whole. Recognising that delirium is often associated with falls, the 4AT has been used to try to reduce falls in some areas as staff can target patients who are at higher risk.

\section{Shared learning}

Elements of the delirium pathway were already being implemented in some test sites, but not in a systematic and formalised way and delirium screening was not previously seen as part of nurses' role. One senior charge nurse commented: 'Take assessment of cognition, for example. We did this but not necessarily for every patient. Now we have a system in place that brings things together.' In this health board the Abbreviated Mental Test tool (Swain and Nightingale 1997) was being used but not routinely, nor within six hours of admission.

In addition to the learnPro modules, education and training have been a major part of efforts to raise awareness of delirium and to engage staff in improving care. Some health boards have run delirium awareness days or weeks where they have held information sessions for colleagues, actively promoting the delirium toolkit. Other boards now run regular training for staff on identifying and managing delirium and others have incorporated delirium into existing education sessions.

Case studies have played an invaluable part in training and helping ward staff understand the value of using the 4AT. Some teams have adapted the 4AT form to include it in patients' notes to record repeat scores or they have incorporated it in their admission process. Elsewhere teams have connected delirium assessment with work on sepsis. Patients who screen positively for sepsis should be started on the Sepsis Six care pathway (Dellinger et al 2008), unless their treatment plan indicates otherwise.

The pathway ensures all appropriate tests are done quickly, allowing rapid diagnosis and treatment of sepsis to reduce the risk of further deterioration.

After comparing the common elements of the Sepsis Six pathway and the delirium work, 4AT has been included in the sepsis pathway as a recognised and reliable tool in some areas. Extending the use of 4AT to a different pathway and different settings has improved monitoring and assessment of acute patients. 
These examples outline some of the results NHS board teams have achieved so far. The programme is changing admission and planning processes, improving engagement with families, reducing falls and length of stay and saving time and resources.

\section{Conclusion}

Benefits of adopting a national approach to improving delirium care include developing a broader perspective, establishing contacts across Scotland and sharing new knowledge and experience. Awareness of delirium has increased and there is evidence of more reliable and consistent processes in delirium screening. The programme has facilitated collaborative working between teams throughout the country and has been augmented by the commitment and engagement of local teams.

With an ageing population and rising numbers of older people admitted to hospital as emergency cases, caring for older people is the core business of acute settings. HIS will continue to work with colleagues in Scotland including the Scottish Delirium Association to ensure a consistent and person-centred approach to the improvement of delirium care for all older people in acute settings.

\section{Implications for practice}

Ensure nursing staff on all levels are aware of the risks of delirium and their role either in preventing it or highlighting concerns to other members of their teams

- Include delirium assessment and documentation of delirium risk as part of nursing assessment and care planning

- Incorporate delirium training as part of educational activities

- Use delirium information leaflets and posters as an introduction to discussions about the risks of delirium with patients, and their families and carers

- Include delirium awareness sessions as part of staff induction

Ask the single question to help identify delirium: 'Is this individual's behaviour the same as it was yesterday?'

Think about how delirium can be avoided by attending to risk factors, such as sensory impairment, pain, dehydration or constipation Engage families in delirium awareness

\section{Online archive}

For related information, visit our online archive and search using the keywords

Conflict of interest None declared

\section{References}

Bellelli G, Morandi A, Davis D et al (2014) Validation of the 4AT, a new instrument for rapid delirium screening: a study in 234 hospitalised older people. Age and Ageing. 43, 4, 496-502.

Dellinger R, Levy M, Carlet J et al (2008) Surviving Sepsis Campaign: international guidelines for management of severe sepsis and septic shock. Intensive Care Medicine. 34, 1, 17-60.

Dewar B, Mackay R, Smith S et al (2010) Use of emotional touchpoints as a method of tapping into the experience of receiving compassionate care in a hospital setting. Journal of Research in Nursing. 15, 1, 29-41.

\section{Fong T, Davis D, Growdon M et al (2015) \\ The interface between delirium and dementia in elderly adults. The Lancet Neurology. $14,8,823-832$ \\ Healthcare Improvement Scotland (2013) Staff, Patients and Families Experiences of Giving and Receiving Care During an Episode of Delirium in an Acute Hospital Care Setting. tinyurl.com/o83cjof (Last accessed: September 23 2015.)}

Healthcare Improvement Scotland (2014a) Think Frailty. Improving the Identification and Management of Frailty: A Case Study Report o Innovation on Four Acute Sites in NHSScotland. HIS, Edinburgh.

\begin{abstract}
Healthcare Improvement Scotland (2014b) Think Delirium. Improving the Care for Older People. Delirium Toolkit. HIS, Edinburgh.

Inouye S, Westendorp R, Saczynski J (2014) Delirium in elderly people. The Lancet. 383, 9920, 911-922.

Langley G, Moen R, Nolan K et al (2009) The Improvement Guide: A Practical Approach to Enhancing Organizational Performance. Second edition. Jossey-Bass, San Francisco CA. MacLullich A, Ryan T, Cash H (2014) 4AT Assessment Test for Delirium and Cognitive Impairment. Version 1.2. www.the4AT.com (Last accessed: September 23 2015.)
\end{abstract}

\author{
National Institute for Health and Care \\ Excellence (2010) Delirium: Diagnosis, \\ Prevention and Management. Clinical \\ Guideline 103. NICE, London. \\ Swain D, Nightingale P (1997) Evaluation of \\ a shortened version of the Abbreviated Mental \\ Test in a series of elderly patients. Clinical \\ Rehabilitation. 11, 3, 243-248. \\ Travers C, Byrne G, Pachana N et al (2013) \\ Delirium in Australian hospitals: \\ a prospective study. Current Gerontology \\ and Geriatrics Research. 2013, 284780. \\ doi:10.1155/2013/284780. \\ Varghese N, Macaden L, Premkumar B et al \\ (2014) Delirium in older people in hospital: \\ an education programme. British Journal of \\ Nursing. 23, 13, 704-709.
}

